\title{
Tandem Spinal Stenosis
}

\author{
A Zulkefli, MS Ortho, R Ramanathan, FRCS \\ Department of Orthopaedic, Hospital Raja Permaisuri Bainun, Ipoh, Malaysia
}

\begin{abstract}
A 42 years old gentleman presented with predominant low back pain associated with bilateral lower limb neurological deficit leading to an initial diagnosis of lumbar stenosis. Further history taking and examination revealed upper limb neurological deficit, and the lower limbs actually presented with upper motor neuron instead of lower motor neuron signs. Imaging studies confirmed the clinical findings with presence of both cervical and lumbar spinal stenosis. Twostage decompression procedures were performed at 6 monthintervals starting with cervical decompression. Postoperative improvement was noted on follow-up. This case highlights the importance of accurate diagnosis of cervical pathology for patients presenting with or referred for predominantly lumbar symptomology.
\end{abstract}

Key Words:

Tandem Spinal Stenosis

\section{INTRODUCTION}

The term 'tandem spinal stenosis' was first introduced by Dagi et al to describe concurrent symptomatic cervical and lumbar spinal stenosis ${ }^{1}$. A typical clinical picture includes intermittent neurogenic claudication, progressive gait disturbance and a combination of myelopathy and polyradiculopathy in both the upper and lower extremities. It is a rather uncommon condition with incidence of $5 \%$ to $25 \%$ of all spinal stenosis cases ${ }^{1}$. Prevalence of tandem spinal stenosis is more common in patients aged 51 years and older $^{2}$. We report a case of tandem spinal stenosis in a 42year-old gentleman.

\section{CASE REPORT}

A 42-years old fisherman was referred to the Orthopaedic Clinic from an outlying clinic with a provisional diagnosis of lumbar spondylosis. He presented with a 6 month history of low back pain following a fall on the buttocks in his boat. The patient's back pain was aggravated by changes in posture. He started to develop bilateral lower limb weakness, which progressively worsened during the initial 3 months following the injury and was associated with a pulling sensation of both lower limbs. At presentation, he had quite an unsteady gait and was only able to walk with a walking frame or personal assistance. There was associated urinary incontinence in the two weeks prior to presenting at the Orthopaedic clinic. There was no bowel incontinence, loss of appetite or weight loss. The lumbosacral spine radiographs taken at the outlying clinic showed loss of lumbar lordosis with multilevel spondylitic changes (Figure 1).

Upon further questioning, the patient not only had lower limb symptoms but also had left upper limb radiating pain with weakness and lower limbs symptom was associated with involuntary jerky motion at times. Examination of the upper limbs demonstrated no objective sensory loss but the patient had loss of motor power to the left hand grip of grade 4. Reflexes were brisk and Hoffman's sign was negative. For the lower limbs, there was significant muscle wasting bilaterally and motor power to the knees and distal points was grade 4. Hyperreflexia was demonstrated at the knee and ankle. Babinski signs were present bilaterally. With the findings of upper motor neuron lesion in the lower limb together with upper limb involvement, the suspicion of simultaneous cervical stenosis was raised and further investigation were conducted. Cervical radiographs showed cervical spondylosis changes with loss of cervical lordosis (Figure 2). MRI of the cervical spine confirmed the presence of multilevel cervical stenosis at $\mathrm{C} 3 \mathrm{C} 4, \mathrm{C} 4 \mathrm{C} 5, \mathrm{C} 5 \mathrm{C} 6$ and $\mathrm{C} 6 \mathrm{C} 7$. The most significant stenosis was at the $\mathrm{C} 6 \mathrm{C} 7$ level (Figure 3). Lumbosacral MRI revealed significant multilevel lumbar spinal stenosis at L2L3, L3L4 and L4L5 levels (with sacralization of L5) (Figure 4).

A diagnosis of tandem spinal stenosis was made. Cervical decompression was carried out first to garner improvement in both the upper and lower limbs. Selective decompression at the $\mathrm{C} 6 \mathrm{C} 7$ level was performed, as this was the level with the most severe stenosis as compared to milder stenosis at higher levels. Then, a $\mathrm{C} 6 \mathrm{C} 7$ discectomy with partial $\mathrm{C} 7$ corpectomy, iliac crest autogenous grafting and $\mathrm{C}$-jaw fixation were performed.

Post-operatively, the patient was followed up at regular intervals. At 6 months post-surgery he showed significant improvement as the left upper limb radicular pain has disappeared, the left hand grip power improved (muscle power 5) and the involuntary jerky lower limb movement had reduced. However the patient had persistent urinary 

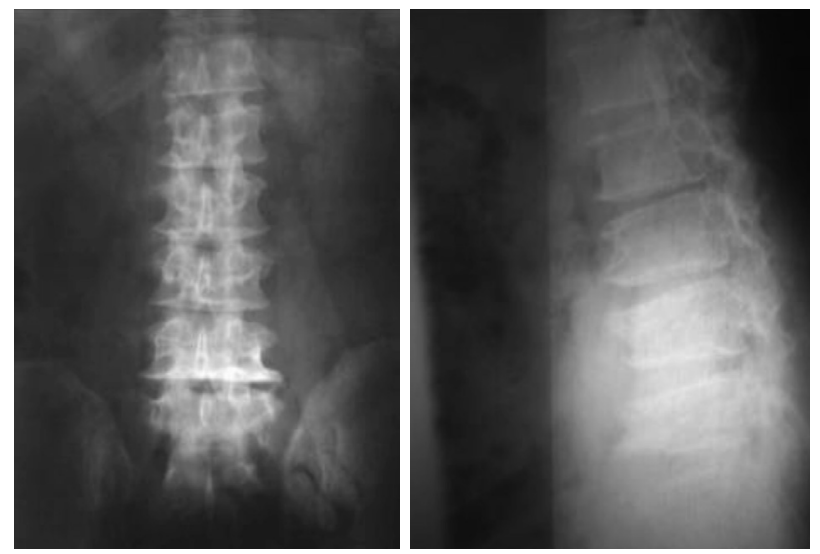

Fig. 1: Radiographs showing lumbar spondylosis changes with loss of sagittal balance (note the sacralization of L5).
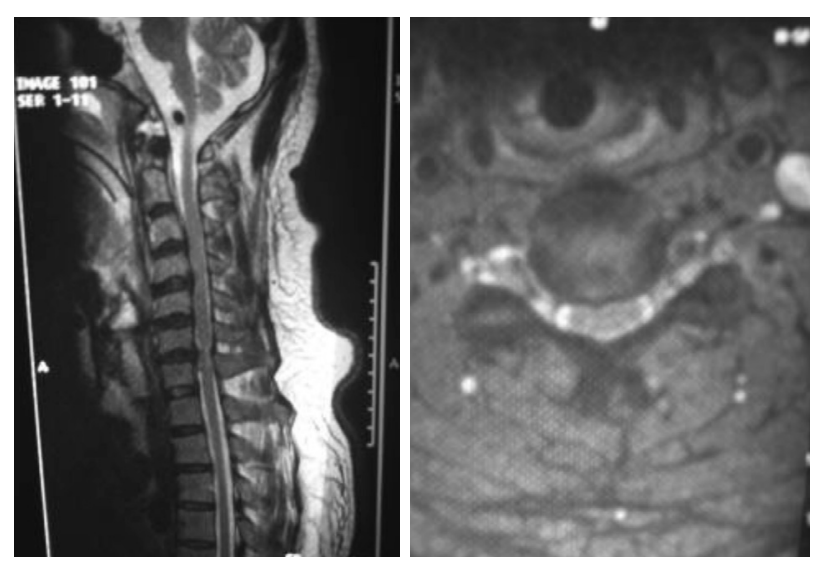

Fig. 3: $\mathrm{MR}$ images showing $\mathrm{C} 6 \mathrm{C} 7$ stenosis with cord changes on sagittal image. Axial image shows flattening of the cord at the same level.
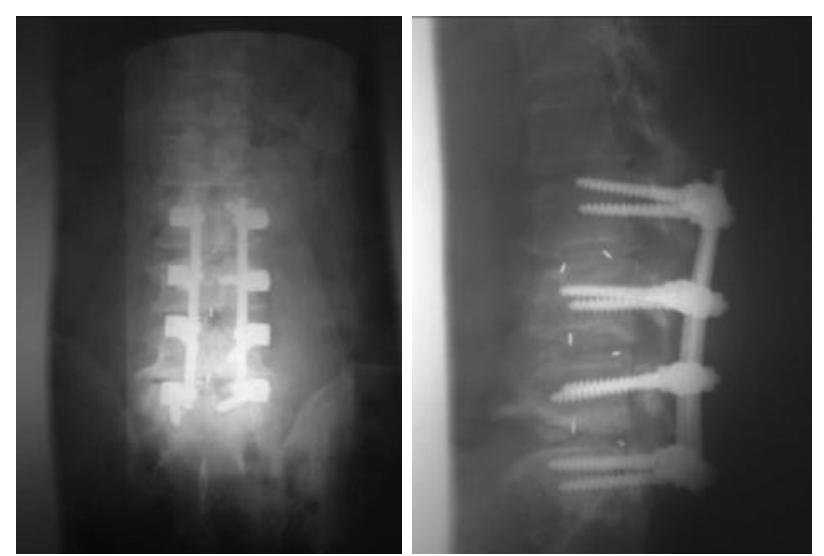

Fig. 5: Radiographs post-operative transforaminal lumbar interbody fusion at L2L3, L3L4 and L4L5.

incontinence, there was no marked improvement of lower limb power and the patient was dependent on a walking frame to ambulate.
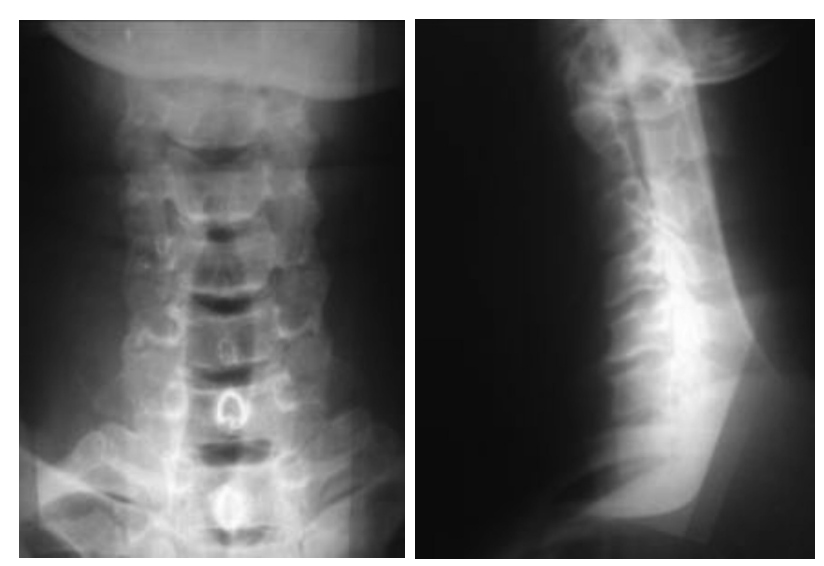

Fig. 2: Radiographs (posteroanterior and Swimmer's views) showing cervical spondylosis changes with loss of sagittal balance (lateral radiograph are not available as the patient has a short neck).
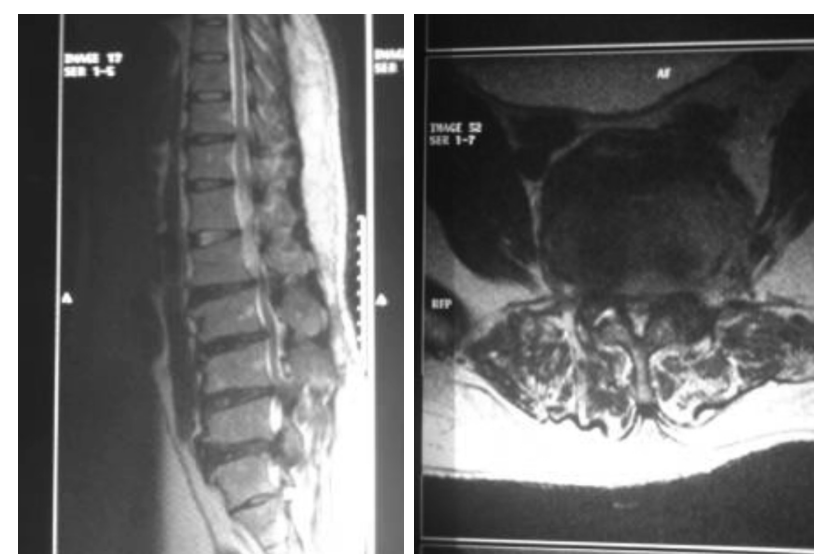

Fig. 4: Lumbosacral MR image showing spinal canal stenosis on sagittal image. Axial image shows a very stenotic canal at L3L4 level.

As the residual neurological deficits were still significant, lumbar decompression was advised. Transforaminal lumbar interbody fusion (TLIF) and decompression at the levels of L2L3, L3L4 and L4L5 was performed 6 months after the cervical operative intervention (Figure 5). Following a postoperative rehabilitation programme, urinary control and the lower limbs strength were improved at 6 months postlumbar decompression surgery. At the most recent follow-up appointment, the Nurick Score had improved from grade 4 pre-operatively (requires someone else's assistance to walk) to grade 3 (difficulty in walking but does not require assistance from anyone to walk). Further follow-up appointments are needed, as the most recent was at less than one year post-surgery, so the final outcome is not yet known.

\section{DISCUSSION}

There are many studies published about spinal stenosis of either the cervical or lumbar regions but very few on concurrent cervical and lumbar spinal stenosis. The association of cervical and lumbar spinal stenosis was first 
reported by Teng and Papatheodorou in $1964^{3}$. They reported 12 cases, 3 of which required both cervical and lumbar laminectomies. In this condition, at initial stage, there may be predominant features of one level over the other, and in fact it is possible that the signs and symptoms of the one level could be obscured by the more prominent features at the second level ${ }^{4,5}$. Therefore the presence of the second level disease might go unrecognized as happened in this case. At the final stage, the clinical presentation of both levels ensues. The features of this type of concurrent stenosis may be confusing as both the upper and lower motor neuron findings are present and both the upper and lower limbs are affected.

Imaging studies are used to confirm the existence and the severity of spinal stenosis at both levels. One must not get confused between tandem spinal stenosis and merely simultaneous existence of radiological spinal stenosis at both the cervical and lumbar levels. Abnormal imaging findings has been reported in 24 to $37 \%$ of asymptomatic cases using varied imaging methodologies including discography, myelography, computed tomography and magnetic resonance imaging ${ }^{4}$. Positive clinical findings should be correlated with the radiological features rather than just relying purely on imaging studies.
Surgical decompression should be performed in indicated cases and among the surgical considerations is which level to be addressed first. Some surgeons prefer to decompress the cervical stenosis first as the cervical decompression might improve lumbar symptom improvement as well while others choose to decompress the more symptomatic level first. Another consideration is whether to decompress one level immediately after the first as two-stage procedure or to decompress both at the same sitting ${ }^{1,4,5}$. Dagi et al found that functional recovery in tandem spinal stenosis depends on early diagnosis and timely surgical intervention ${ }^{1}$. Out of 19 cases in their series, $26 \%$ has an excellent outcome, $21 \%$ improved, 26\% deteriorated despite initial improvement and one patient was unchanged.

One important learning point in this case report is not to overlook the potential presence of tandem spinal stenosis when a patient presents with either predominant cervical or lumbar spinal stenosis features. Failure to detect cervical stenosis in a patient that presents with predominant lumbar stenosis as illustrated in this case report, may carry significant risk of injury to the cervical cord during positioning for lumbar decompression surgery. Detail history taking and thorough physical examination is the key to excluding this condition. 


\section{REFERENCES}

1. Dagi TF, Trakington MA, Leech JJ. Tandem lumbar and cervical spine stenosis. Natural history, prognostic indices, and results after surgical decompression. J Neurosurg. 1987; 66: 842-9.

2. LaBan MM. Green ML. Concurrent (tandem) cervical and lumbar spinal stenosis: a 10-yr review of 54 hospitalized patients. $A m$ J Phys Med \& Rehabil, 2004; 83(3): 187-90.

3. Teng P, Papatheodorou C. Combined cervical and lumbar spondylosis. Arch Neurol. 1964; 10: 298-308.

4. Naderi S, Mertol T. Simultaneous cervical and lumbar surgery for combined symptomatic cervical and lumbar spinal stenosis. J Spinal Disord Tech, 2002; 15: 229-32.

5. Aydogan M, Ozturk C, Mirzanli C, Karatoprak O, Tezer M, Hamzaoglu A. Treatment approach in tandem (concurrent) cervical and lumbar spinal stenosis. Acta Orthop Belg. 2007; 73(2): 234-7. 